\title{
Microbial taxa in dust and excreta associated with the productive performance of commercial meat chicken flocks
}

Yugal Raj Bindari ${ }^{1}$, Robert J. Moore ${ }^{2}$, Thi Thu Hao Van², Stephen W. Walkden-Brown ${ }^{1}$ and Priscilla F. Gerber ${ }^{1^{*}}$ (D)

\begin{abstract}
Background: A major focus of research on the gut microbiota of poultry has been to define signatures of a healthy gut and identify microbiota components that correlate with feed conversion. However, there is a high variation in individual gut microbiota profiles and their association with performance. Population level samples such as dust and pooled excreta could be useful to investigate bacterial signatures associated with productivity at the flock-level. This study was designed to investigate the bacterial signatures of high and low-performing commercial meat chicken farms in dust and pooled excreta samples. Poultry house dust and fresh pooled excreta were collected at days 7, 14, 21,28 and 35 of age from 8 farms of two Australian integrator companies and 389 samples assessed by 165 ribosomal RNA gene amplicon sequencing. The farms were ranked as low $(n=4)$ or high performers $(n=4)$ based on feed conversion rate corrected by body weight.

Results: Permutational analysis of variance based on Bray-Curtis dissimilarities using abundance data for bacterial community structure results showed that company explained the highest variation in the bacterial community structure in excreta $\left(R^{2}=0.21, p=0.001\right)$ while age explained the highest variation in the bacterial community structure in dust $\left(R^{2}=0.13, p=0.001\right)$. Farm performance explained the least variation in the bacterial community structure in both dust $\left(R^{2}=0.03, p=0.001\right)$ and excreta $\left(R^{2}=0.01, p=0.001\right)$ samples. However, specific bacterial taxa were found to be associated with high and low performance in both dust and excreta. The bacteria taxa associated with high-performing farms in dust or excreta found in this study were Enterococcus and Candidatus Arthromitus whereas bacterial taxa associated with low-performing farms included Nocardia, Lapillococcus, Brachybacterium, Ruania, Dietzia, Brevibacterium, Jeotgalicoccus, Corynebacterium and Aerococcus.
\end{abstract}

Conclusions: Dust and excreta could be useful for investigating bacterial signatures associated with high and low performance in commercial poultry farms. Further studies on a larger number of farms are needed to determine if the bacterial signatures found in this study are reproducible.

Keywords: Chicken, Microbiota, Performance, Signatures, Dust, Excreta

*Correspondence: pgerber2@une.edu.au

${ }^{1}$ Animal Science, School of Environmental and Rural Science, University of New England, Armidale, NSW 2351, Australia

Full list of author information is available at the end of the article

\section{Introduction}

The taxonomic composition of gut microbiota of poultry is affected by various factors such as diet, environment, breed, infectious agents, and management, and has been shown to influence bird health, nutrition, and physiology [1-4]. Methods for monitoring of gut health, which can be defined as the 'state of symbiotic equilibrium between the microbiota and intestinal tract where 
animal health and welfare remains unaltered due to dysfunctional intestine' is a prime focus in poultry research as a healthy gut is required to ensure bird welfare and to prevent production losses [5]. Several experimental studies have shown that differentially abundant bacterial taxa identified in excreta, ileum, and caecum contents, can be correlated with either good or poor bird production performance [6-8]. Specifically, bacterial groups that are commonly known as butyrate producers and resistant starch degraders are usually associated with increased bird feed efficiency $[8,9]$. However, the bacterial composition of the gut microbiota of individual birds is highly variable within and between studies and specific bacterial signatures that reliably correlate with good or poor flock performance remain elusive $[9,10]$.

Within the chicken gut, the highest microbial density and the most complex microbial community is found in the caecum, and therefore caecal content has been commonly used to investigate the gut microbiota [8]. The collection of caecum or gut contents has some drawbacks as it requires birds to be euthanized and precludes longitudinal studies in the same birds [11]. Therefore, there is a need for the development of methods that use noninvasive samples to evaluate gut microbiota [11, 12]. Previous studies evaluating excreta, boot sock samples and caecal droppings have suggested that boot sock samples and caecal droppings could be useful alternatives to study caecal microbiota composition longitudinally $[11,13]$. The rationale for the use of these alternative sample types is that they are derived from the gastrointestinal tract of chickens and could therefore be used to infer gut health and perhaps performance. However, the relationship of the microbiota composition using noninvasive population level samples and flock performance is not well understood. As measurements of productive performance and management interventions in commercial meat chicken farms are mostly applied at farmlevel, a monitoring strategy at a population-level would perhaps be advantageous compared to sampling of individual birds. The use of non-invasive population level samples such as poultry dust, pooled excreta, and litter has several advantages compared to individual sampling of birds, such as ease of sample collection and the use of a small number of samples to represent a population. Dust samples, in particular, are dry, stable and can be shipped at room temperature. A recent study has shown that genomic material of RNA and DNA viruses was stable in poultry dust for at least to 4 months when stored at temperatures up to $37^{\circ} \mathrm{C}[14]$.

In this study, poultry house dust and fresh pooled excreta from the floor were collected weekly from eight commercial meat chicken farms of two Australian integrator companies. The farms were ranked as low or high performers based on feed conversion ratio corrected for body weight by the integrator companies. We hypothesized that microbiota profiles of pooled excreta and dust collected from poultry farms would provide specific bacterial signatures associated with high and low-productive farm performance. This study was specifically designed to (1) Determine and compare the bacterial taxa of poultry dust and pooled excreta in the two integrator companies; (2) Determine if the bacterial taxa in dust and pooled excreta could differentiate high and low-performing farms.

\section{Methodology}

\section{Farms and samples collection}

The study was conducted on 16 flocks from eight commercial meat chicken farms $(n=2$ flocks/farm) in a $2 \times 2 \times 5$ factorial arrangement with two Australian integrator companies (A and B), two levels of farm productive performance (high and low), and five sampling times $(7,14,21,28$ and 35 days of chicken age) as previously described [15]. Details of the farms are included in Additional file 1. Samples were collected between August 26 and November 19, 2019. The integrator companies varied in a number of management practices, including the choice of chicken strain, geographic location, and feed formulation. All farms from company A were located in the outskirts of Sydney in New South Wales, used the Cobb strain of chickens and used a range of bedding materials (wood shavings, sawdust, biobedding) (Additional file 1). All farms from company B were located in the outskirts of Adelaide, South Australia, used the Ross strain of chickens and straw as bedding material. Two farms with consistent high performance and two farms with consistent low performance based on historical production data were selected by veterinarians from each company. The productive performance ranking of each farm, which is based on the corrected feed conversion rate for the whole farm, was then recorded at the end of the production cycle which confirmed that the studied flocks ranked high (above the 50 percentile of production) or low (below the 50 percentile). Pooled excreta and dust samples were collected weekly from two poultry houses in each farm at 7, 14, 21, 28 and 35 days of the production cycle. Settled dust samples were collected using two funnels held in an apparatus suspended at $1.5 \mathrm{~m}$ height on the wires in the house that support feeders and waterers. The funnels which were put in place on the day of chick placement captured setting dust and directed it into a collection vial attached to the funnel that was removed at each sampling and replaced with a new vial. Settled dust samples were dry and with fine consistency and submitted vials had enough material for testing (at least $10 \mathrm{mg}$ of dust). Five fresh individual excreta samples 
were collected from the poultry house floor to form one pooled sample and four pooled excreta samples were collected from each poultry house in a stratified sampling pattern. A total of 406 samples (dust $=138$, excreta $=268$ ) were received from the participant farms, from which 17 samples were discarded because of poor quality DNA making a total of 389 samples analysed in this study (dust $=131$; excreta $=258$ ).

\section{DNA extraction}

Previous experience with these types of samples had shown that no one DNA extraction method gave good DNA yields from both dust and excreta. To maximise DNA quality and yields from each sample type different extraction kits were used. DNA extraction of dust was performed using the QIAamp ${ }^{\circledR}$ Fast DNA Stool Mini Kit (Qiagen, Hilden, Germany) according to the manufacturer's instructions with minor modifications [15]. Briefly, $0.4 \mathrm{~g}$ of $1 \mathrm{~mm}$ glass beads were added to $2 \mathrm{ml}$ microtubes containing $1 \mathrm{ml}$ of InhibitEX and $10 \mathrm{mg}$ of dust and homogenised for 5 min at maximum speed using a Qiagen Tissue Lyser II (Qiagen, Hilden, Germany). The homogenised suspensions were then heated at $95^{\circ} \mathrm{C}$ for 10 min. DNA extraction from excreta was performed using the DNeasy PowerSoil Pro Kit (Qiagen, Hilden, Germany) according to the manufacturer's instructions, except that $50 \mathrm{mg}$ of excreta were homogenised for $5 \mathrm{~min}$ with PowerBead at maximum speed using Qiagen Tissue Lyser II (Qiagen, Hilden, Germany) with subsequent heating of homogenised suspensions at $90{ }^{\circ} \mathrm{C}$ for $10 \mathrm{~min}$. It is acknowledged that different extraction methods can result in different $16 \mathrm{~S}$ amplicon analysis results [16]. The type of sample and DNA extraction kits affect the quality of DNA and composition of bacteria following 16S rRNA gene sequencing using Illumina MiSeq sequencer [16, 17] but such differences are mainly restricted to the yield of Gram-positive bacterial DNA due to efficiency of cell breakage. Both methods used in the current study used efficient bead-beating protocols to minimise this effect.

\section{$16 \mathrm{~S}$ rRNA gene amplification and analysis}

Microbiota composition was assessed by sequencing of amplicons across the V3-V4 region of 16S rRNA genes. Amplicons were produced using custom-designed barcoded primers targeting the 343-806 region, ACTCCT ACGGGAGGCAGCAG (forward primer) and GGA CTACHVGGGTWTCTAAT (reverse primer), primers also contained spacer sequences and Illumina sequencing linkers, following the design of Fadrosh et al. [18]. The amplicons were sequenced on an Illumina MiSeq Sequencer using $2 \times 300$ bp paired-end reads. Sequence data were trimmed with Trimmomatic and then fatsq files were analysed using DADA2 in QIIME2 v2020.6 [19] to denoise and produce Amplicon Sequence Variants (ASVs). ASVs were clustered at 99\% identity using the VSEARCH plugin [20]. Taxonomy was assigned using the SILVA database. All of the downstream statistical microbial data analysis and visualisation were done using Calypso software [21]. A total of 9624 ASVs were found. The sequence data used for analysis is available in NCBI under BioProject accession number PRJNA730489.

\section{Statistical analysis}

The bacterial community composition data was normalised using Hellinger transformation before statistical comparison. Statistical analyses were performed using the Calypso software (http://cgenome.net/wiki/ index.php/Calypso) except for the permutational multivariate analysis of variance (PERMANOVA) which was performed using the PRIMER v7 software including the PERMANOVA + add-on module [22].

Distance based redundancy analysis (dbRDA) using Bray-Curtis dissimilarity was used to visualize the difference in bacterial community structure between companies in dust and excreta and principal-coordinate analysis (PCoA) using Bray-Curtis dissimilarity was used to visualize the differences in bacterial community structures between bird age (7, 14, 21, 28 and 35 days). To examine the effects of sample type, company, bird age, performance and their interactions in the bacterial community structures, permutational multivariate analysis of variance (PERMANOVA) based on BrayCurtis dissimilarities using the default setting of Type III sum of squares was used [23]. Homogeneity of multivariate dispersion was tested using permutational analyses of multivariate dispersions (PERMDISP) [24]. Linear discriminant analysis effect size (LEfSe) was used to test the differences in abundance of the bacterial taxa between companies (A and $\mathrm{B}$ ). The top 50 most abundant taxa were selected and Wilcoxon test, area under the curve (AUC) and odds ratio (OR) were used to identify bacterial taxa more prevalent in high and low-performing farms. $P$ values were corrected with the false discovery rate and adjusted $p$ value $(\mathrm{q})<0.05$ was considered statistically significant. Bacterial taxa with significantly different $(\mathrm{q}<0.05)$ abundance in high and low-performing farms with an AUC $\geq 0.90$ were subjected for further OR analysis. The higher likelihood of bacterial taxa occurring in a certain production performance category (high, low) (AUC $\geq 0.90$, Wilcoxon test $\mathrm{q}$ value $<0.05$ ) were considered as 'signatures' of that production outcome. 


\section{Results}

Company and age explained the most variation in the structure of bacterial community in pooled excreta and dust while performance explained the least variation in both sample types

As indicated in material and methods two different methods were used for DNA extraction of dust and excreta which could add variability on the detected bacterial communities. Therefore, data was analysed by PERMANOVA using model accounting to all variables in the study (sample type, company, age and performance) to assess if the microbial taxa was consistent between samples types and also for each sample type separately.

When analysing the whole model, sample type explained the most variation in the bacterial community structure (12\%), followed by company (6\%), and bird age (5\%), while farm performance explained the least variation $(1 \%)$ with significant interaction among these factors (Additional files 2; 3).

In dust samples, age explained the most variation in the bacterial community structure (13\%), followed by company (11\%) and the least variation was explained by performance (3\%), with significant interactions among these factors (Table 1). In excreta, company explained most of the variation (21\%) followed by bird age $(10 \%)$ and performance (1\%) with significant interactions among these factors (Table 1 ).

\section{Sample type}

Taxonomic assignment at genus level of the bacteria found in dust and excreta are shown in Fig. 1. The most dominant bacterial genera found in excreta of company A were Weissella (mean percentage \pm SD $0.38 \pm 0.15$ ) and Lactobacillus $(0.24 \pm 0.17)$ while in company B were Lactobacillus $(0.53 \pm 0.20)$ and Corynebacterium $(0.10 \pm 0.08)$ (Fig. 1). In dust samples, Brevibacterium $(0.22 \pm 0.11)$ and Brachybacterium $(0.13 \pm 0.09)$ were the most dominant bacterial genera in company $\mathrm{A}$ while in company B were Staphylococcus $(0.15 \pm 0.08)$, Corynebacterium $(0.12 \pm 0.05)$ and Brachybacterium $(0.12 \pm 0.08)$ (Fig. 1). At phylum level, the most dominant bacterial phyla in excreta and dust of company A and B were Firmicutes $(0.38 \pm 0.24-0.85 \pm 0.13)$ and Actinobacteria $(0.14 \pm 0.13-0.50 \pm 0.24)$ (Additional file 4$)$. Of the bacterial taxa (genus level) detected in excreta of company A or B, $81 \%$ were also detected in dust. The shared bacterial genera between dust and excreta and the bacterial genera that are exclusive to dust or excreta of companies $A$ and $B$ are shown in Additional files 5 and 6.

Microbial richness determined by Chao1 diversity index was higher in dust compared to excreta in both companies $(p<0.001)$ (Fig. 2). The bacterial genera that differentiate dust and excreta of companies $\mathrm{A}$ and $\mathrm{B}$ shown by LEfSe are presented in Additional file 7 .

\section{Company}

In dust and excreta, $55 \%$ and $52 \%$ of the bacterial genera were shared between companies, respectively. The

Table 1 Permutational multivariate analysis of variance (PERMANOVA) results showing the influence of company, age, performance and their interactions on the bacterial community structure in excreta and dust samples

\begin{tabular}{|c|c|c|c|c|c|c|c|}
\hline Sample type & Source & df & Sum of squares & Pseudo-F & $\mathrm{R}^{2}$ & $P$ value & PERMDISP $P$ value \\
\hline \multirow[t]{8}{*}{ Excreta } & Company & 1 & 167,120 & 91.10 & 0.21 & 0.001 & $<0.001$ \\
\hline & Age & 4 & 81,418 & 11.10 & 0.10 & 0.001 & 0.008 \\
\hline & Performance & 1 & 10,863 & 5.92 & 0.01 & 0.001 & 0.03 \\
\hline & Age $\times$ company & 4 & 39,511 & 5.38 & 0.05 & 0.001 & \\
\hline & Company $\times$ performance & 1 & 12,723 & 6.94 & 0.02 & 0.001 & \\
\hline & Age $\times$ performance & 4 & 14,605 & 1.99 & 0.02 & 0.001 & \\
\hline & Performance $\times$ age $\times$ company & 4 & 19,093 & 2.60 & 0.02 & 0.001 & \\
\hline & Residuals & 238 & & & & & \\
\hline \multirow[t]{8}{*}{ Dust } & Company & 1 & 29,643 & 21.94 & 0.11 & 0.001 & 0.003 \\
\hline & Age & 4 & 35,962 & 6.65 & 0.13 & 0.001 & $<0.001$ \\
\hline & Performance & 1 & 9151.6 & 6.77 & 0.03 & 0.001 & 0.11 \\
\hline & Age $\times$ company & 4 & 15,488 & 2.87 & 0.06 & 0.001 & \\
\hline & Company $\times$ performance & 1 & 4559.6 & 3.37 & 0.02 & 0.001 & \\
\hline & Age $\times$ performance & 4 & 8810.5 & 1.63 & 0.03 & 0.001 & \\
\hline & Performance $\times$ age $\times$ company & 4 & 11,313 & 2.09 & 0.04 & 0.001 & \\
\hline & Residuals & 111 & & & & & \\
\hline
\end{tabular}



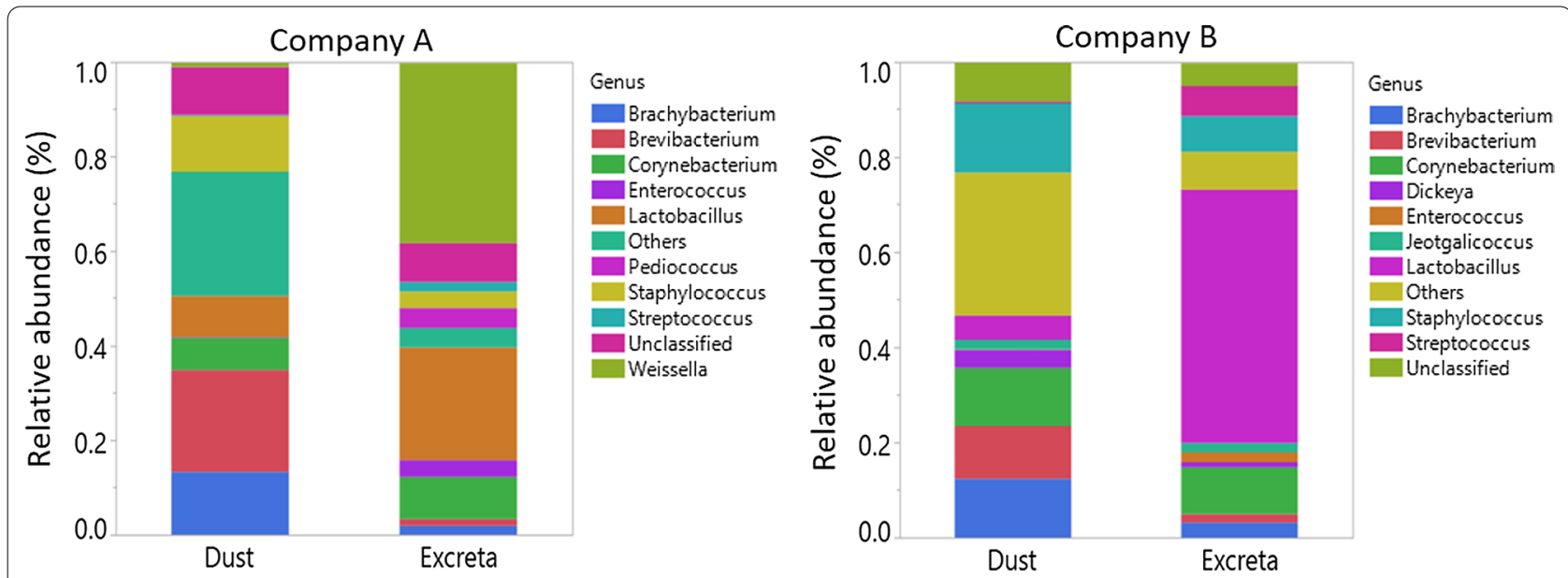

Fig. 1 Taxonomic assignment of the bacteria at genus level in dust and excreta stratified by company. The levels of the top 10 most abundant genera were shown. Genera that were not included under top 10 most abundant ones were merged together and presented as 'Others'
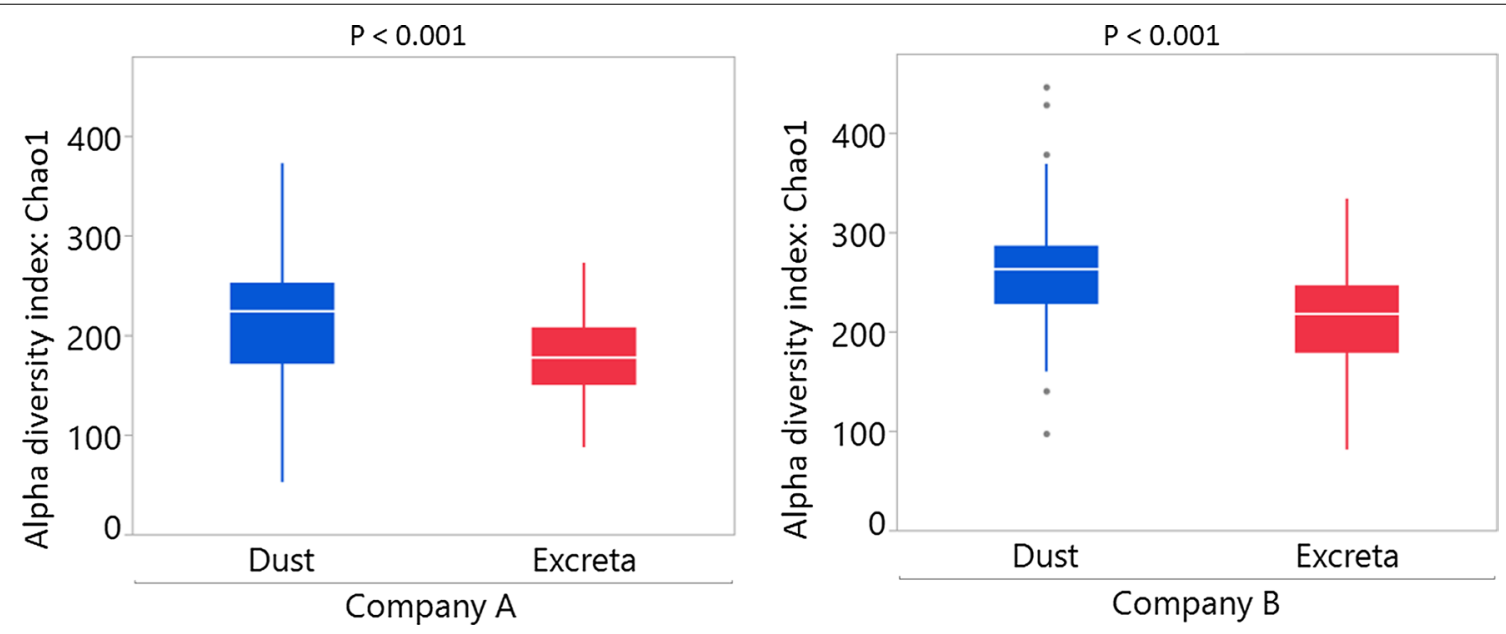

Fig. 2 Microbial richness estimated using Chaol diversity index for dust and excreta of companies A and B. All ages were combined for the analysis

shared bacterial genera between companies and the bacterial genera that are exclusive to companies $\mathrm{A}$ and $\mathrm{B}$ are shown in Additional files 8 and 9. Within each company, microbial communities of pooled excreta (PERMANOVA $p=0.001$ and $\mathrm{R}^{2}=0.23$; PERMDISP $\left.p<0.001\right)$ were more dissimilar than dust (PERMANOVA $p=0.001$ and $\mathrm{R}^{2}=0.12$; PERMDISP $p=0.003$ ) which was also evident in the redundancy analysis ordination plot (Fig. 3). The bacterial genera that differentiate companies A and B in dust and excreta are shown in Additional file 10.

\section{Bird age}

As expected, age had a significant effect on the bacterial community structure in both dust and excreta. In excreta, age explained $16 \%$ variation in company
A (PERMANOVA $p=0.001$ ) and $24 \%$ in company B $(p=0.001)$ while in dust age explained $19 \%$ variation in company A $(p=0.001)$ and $32 \%$ in company $\mathrm{B}(p=0.001)$.

Bacterial richness was lower at day 7 in company $\mathrm{A}$ and B farms in excreta (Fig. 4a) and this age group was the most divergent compared to samples collected afterwards (Fig. 4b) when bacterial richness stabilised. Similar results were seen in dust of company B farms while in company A farms bacterial richness was found to be similar across all ages. Bacterial richness was higher in dust compared to excreta in company A except at days 21 and 28 when dust and excreta had similar microbial richness (Fig. 4a). Similarly, in company B, bacterial richness was higher in dust compared to excreta except at days 7 and 14 when microbial richness between dust and 

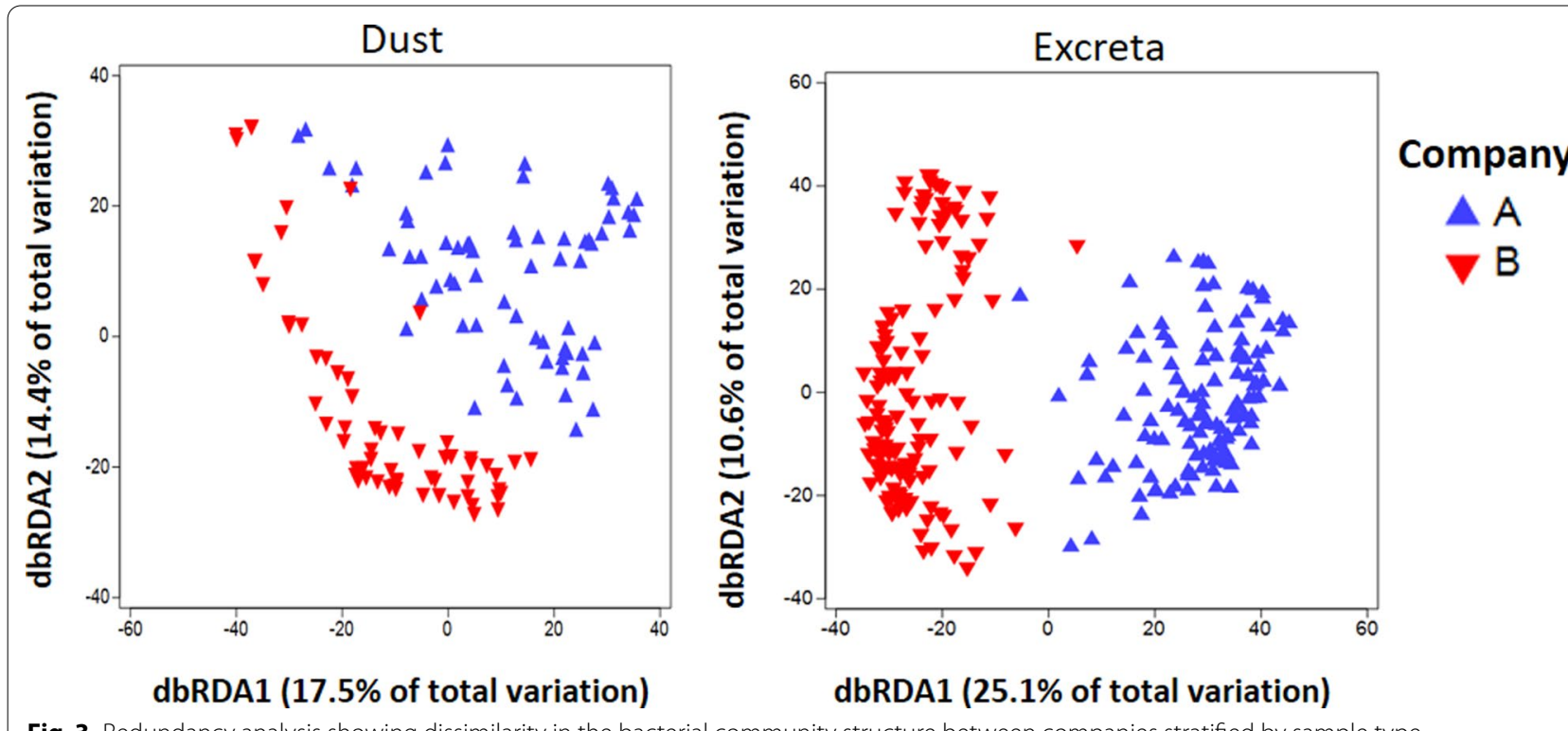

Fig. 3 Redundancy analysis showing dissimilarity in the bacterial community structure between companies stratified by sample type

excreta were similar (Fig. 4a). Figure 5 shows the relative abundance of bacterial genera across different ages in dust and excreta samples of company A and B. From days 7-35, excreta were dominated by Weissella in company A and Lactobacillus in company B. Dust was dominated by Unclassified bacterial groups at day 7 and Brevibacterium at days 14-35 in company A while in company B dust was dominated by Unclassified bacterial taxa at day 7, Staphylococcus at days 14-21, Corynebacterium and Brevibacterium at days 28 and 35.

\section{Performance}

Production explained the least variation within the dataset in both dust and excreta (Table 1). PERMANOVA results showed highest variation in the bacterial community structure between high and low production farms in dust at day 7 for company A and at day 28 for company B while in excreta it was at day 7 for company A and day 14 for company B (Table 2).

\section{Bacterial signatures of low and high-performing farms}

Although the farm performance explained the least variation in the bacterial community structure, some bacterial taxa were overrepresented in low or high performing farms and met the 'bacterial signature' criteria (AUC $\geq 0.90$, Wilcoxon test q value $<0.05$ ) (Figs. 6, 7, 8, 9; Additional files 11, 12, 13, 14).

\section{Dust samples}

No bacterial taxa met the criteria for bacterial signature in high-performing farms of company A; whereas for company B Dickeya (day 35) met the bacterial signature criteria (Figs. 6, 7). Although not statistically significant, the abundance of Dickeya was also numerically higher in high-performing farms of company A at day 28.

Bacterial taxa in dust of low-performing farms of company A that meet the bacterial signature criteria were Nocardia (day 21), Lapillococcus (day 21), Brachybacterium (day 21), Ruania (days 21 and 28), Dietzia (day 21) and Brevibacterium (day 21); whereas for company B were Lapillicoccus (day 35) and Ruania (day 35) (Figs. 6, 7). Although not statistically significant, the abundance of Brachybacterium and Dietzia were also observed numerically at a higher level in low-performing farms for company A at days 14 and 28, Ruania at days 14, 21 and 35 and Brevibacterium at days 7, 14, 28 and 35; whereas in company B the abundance of Lapillococcus and Runia were numerically higher at days 7 to 28 .

\section{Excreta}

Bacterial taxa that meet the bacterial signature criteria of high-performing farms in company A were Enterococcus (day 7) and unclassified group of bacterial taxa (day 7); whereas for company B were Enterococcus (day 35) and Candidatus Arthromitus (day 14) (Figs. 8, 9). Although not statistically significant, the abundance of unclassified group of bacterial taxa and Enterococcus were also observed numerically at a higher level in high-performing farms of company A at days 14-28; while for company B was Candidatus Arthromitus at days 7, 21 and 28.

No bacterial taxa meet the bacterial signature criteria of low performing farms for company A; whereas for 


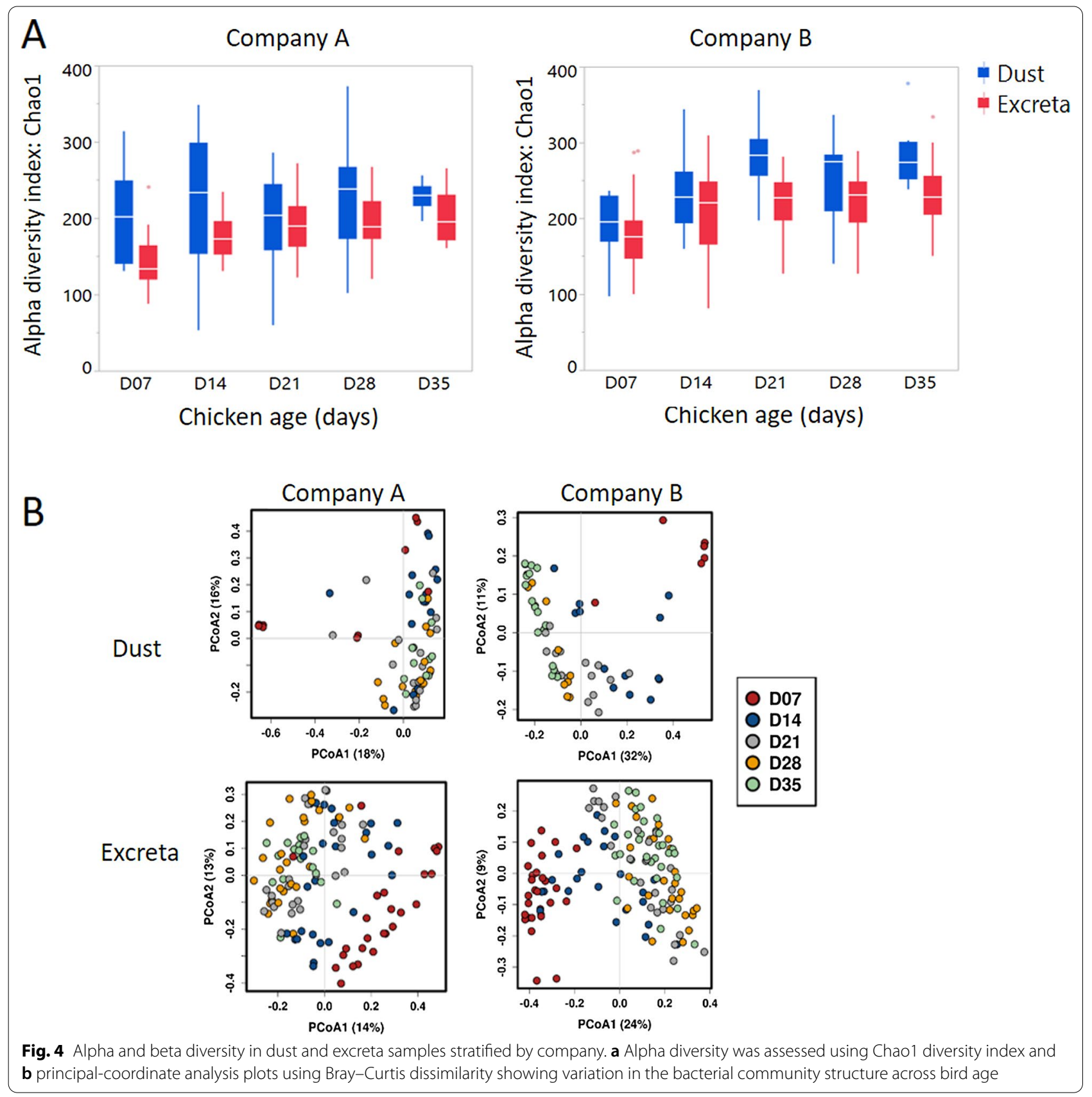

company B bacterial taxa that meet the bacterial signature criteria were Jeotgalicoccus (day 14), Corynebacterium (day 14) and Aerococcus (day 14) (Figs. 8, 9). Although not statistically significant, the abundance of Jeotgalicoccus was also observed numerically at a higher level in low-performing farms of company B at days 7, 21 and 28, and Corynebacterium and Aerococcus at days 21 and 28. There was no overlap in the bacterial signatures from high and low-performing farms between dust and excreta of company A and B farms (Figs. 6, 7, 8, 9).

\section{Discussion}

Many studies have associated gut microbiota with feed conversion and weight gain in meat chickens under experimental conditions. We therefore hypothesized that microbiota profiles of population level samples would identify specific bacterial taxa associated with productive performance in commercial meat chicken farms. In the current study, overall farm performance explained the least amount of variation in the bacterial community structure for both dust and excreta samples. Although 

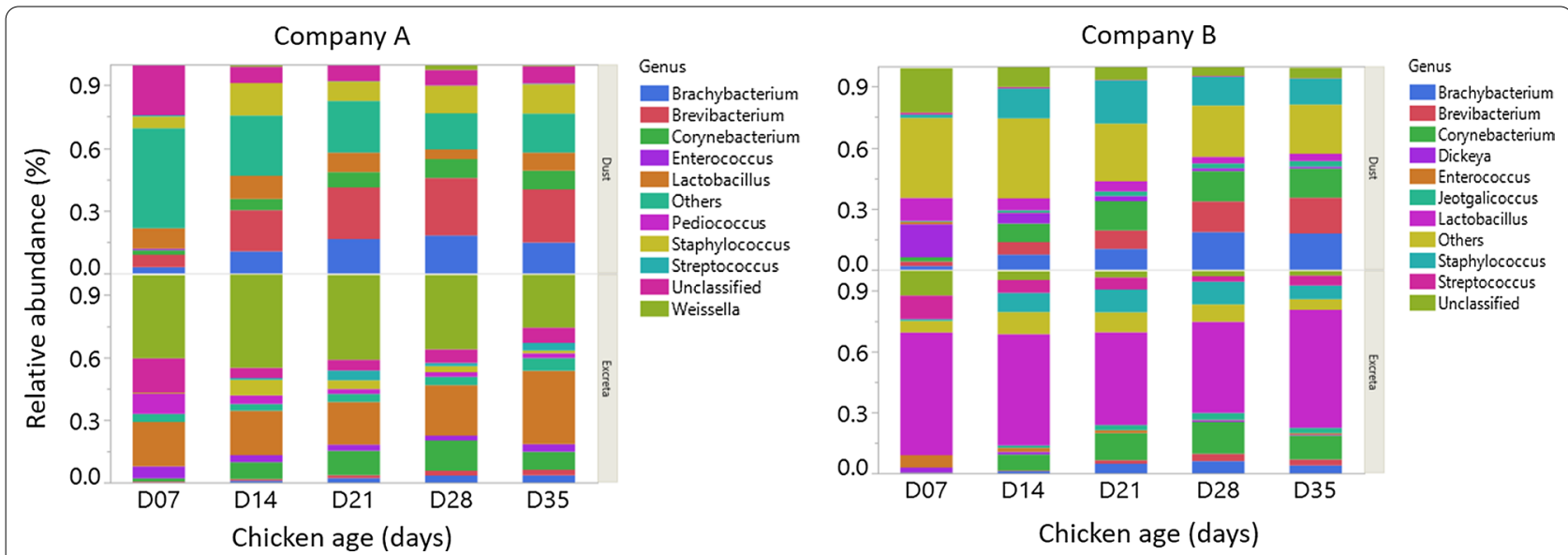

Fig. 5 Top 10 most abundant taxa of company A and B farms in dust and excreta samples across different age of the chickens $(7,14,21,28$ and 35$)$. Genera that were not included under top 10 most abundant ones were merged together and presented as 'Others'

Table 2 Variation in the bacterial community structure between high and low-performing farms of company A and B across the production cycle (7, 14, 21, 28 and 35) in excreta and dust samples using permutational multivariate analysis of variance (PERMANOVA)

\begin{tabular}{|c|c|c|c|c|c|c|}
\hline \multirow[t]{2}{*}{ Sample type } & \multirow[t]{2}{*}{ Company } & \multirow[t]{2}{*}{ Chicken Age (days) } & \multicolumn{3}{|c|}{ PERMANOVA } & \multirow{2}{*}{$\begin{array}{l}\text { PERMDISP } \\
P \text { value }\end{array}$} \\
\hline & & & Pseudo-F & $\mathrm{R}^{2}$ & $P$ value & \\
\hline \multirow[t]{12}{*}{ Excreta } & $A$ & All ages & 6.84 & 0.05 & 0.001 & 0.33 \\
\hline & & 7 & 6.03 & 0.20 & 0.001 & 0.90 \\
\hline & & 14 & 3.49 & 0.13 & 0.003 & 0.87 \\
\hline & & 21 & 2.83 & 0.11 & 0.004 & 0.65 \\
\hline & & 28 & 3.16 & 0.12 & 0.001 & 0.16 \\
\hline & & 35 & 2.17 & 0.13 & 0.007 & 0.81 \\
\hline & B & All ages & 3.39 & 0.02 & 0.003 & 0.97 \\
\hline & & 7 & 1.43 & 0.05 & 0.10 & 0.62 \\
\hline & & 14 & 4.67 & 0.18 & 0.001 & 0.14 \\
\hline & & 21 & 2.01 & 0.08 & 0.03 & 0.19 \\
\hline & & 28 & 2.77 & 0.10 & 0.001 & 0.36 \\
\hline & & 35 & 1.40 & 0.04 & 0.10 & 0.77 \\
\hline \multirow[t]{11}{*}{ Dust } & A & All ages & 5.54 & 0.08 & 0.001 & 0.02 \\
\hline & & 7 & 2.65 & 0.25 & 0.08 & 0.75 \\
\hline & & 14 & 2.83 & 0.18 & 0.001 & 0.61 \\
\hline & & 21 & 2.78 & 0.17 & 0.002 & 0.07 \\
\hline & & 28 & 1.97 & 0.12 & 0.04 & 0.004 \\
\hline & & 35 & 2.47 & 0.22 & 0.007 & 0.005 \\
\hline & B & All ages* & 2.97 & 0.05 & 0.01 & 0.48 \\
\hline & & 14 & 1.78 & 0.14 & 0.11 & 0.02 \\
\hline & & 21 & 1.66 & 0.11 & 0.07 & 0.21 \\
\hline & & 28 & 3.22 & 0.24 & 0.009 & 0.003 \\
\hline & & 35 & 2.11 & 0.13 & 0.02 & 0.08 \\
\hline
\end{tabular}

PERMDISP, permutational analyses of multivariate dispersions

*Dust samples collected on day 7 from company B were excluded due to the small sample

Values in bold indicate statistical significance $(p<0.05)$ 


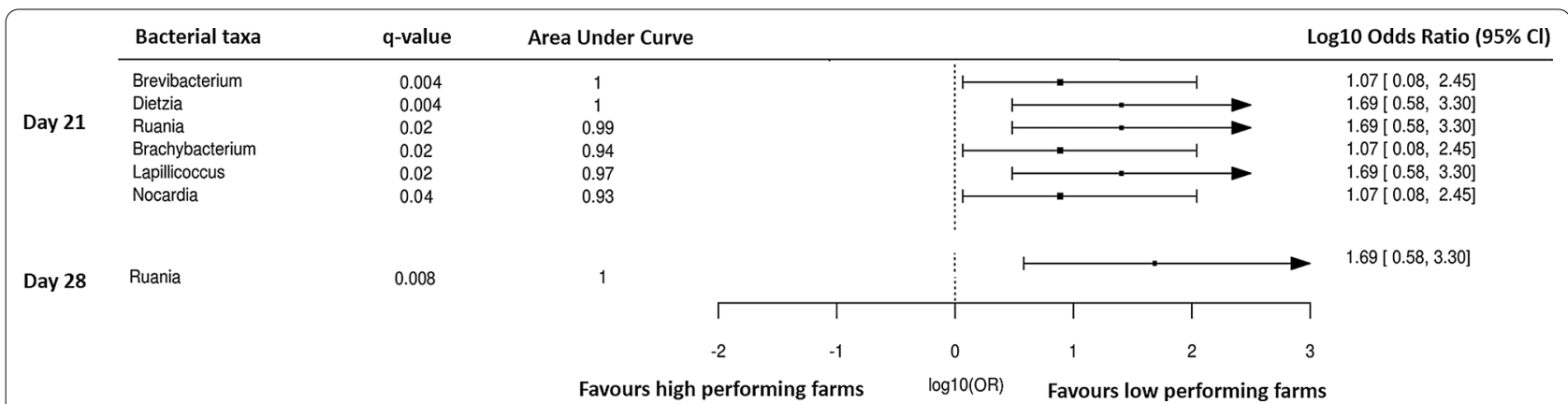

Fig. 6 Forest plot of the significantly different bacterial taxa (Wilcoxon q value $<0.05$ ) of company A farms in dust with AUC $\geq 90$. The results to the left of the vertical dotted line (line of no effect) denote the increased chance of occurrence of microbiota towards high-performing farm and the results to the right of the vertical dotted line denotes the increased chance of occurrence of microbiota towards the low-performing farm

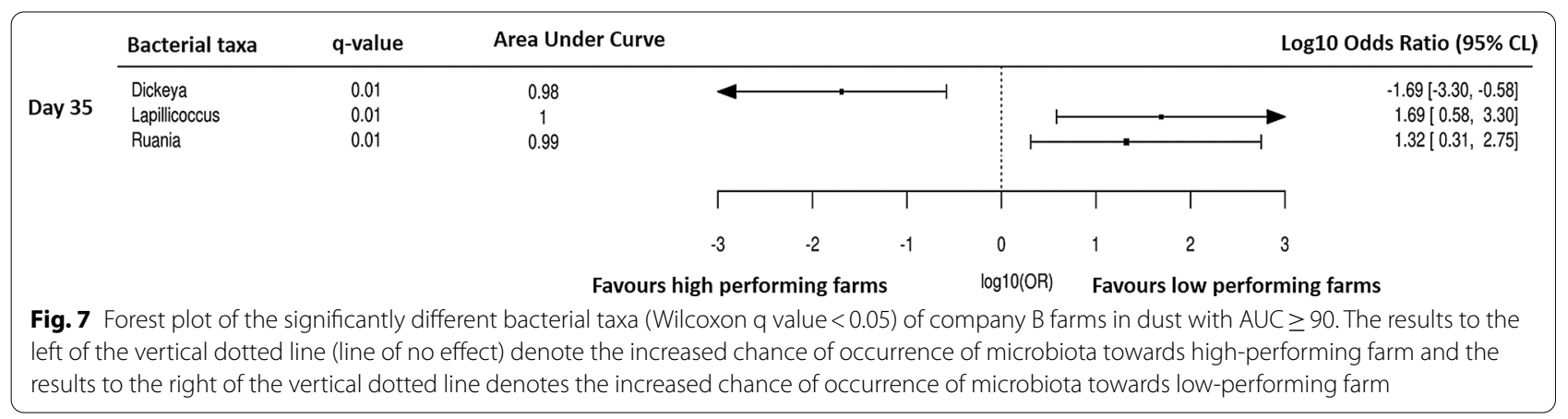

\begin{tabular}{|llll|l|l|}
\hline Day 7 & Bacterial taxa & q-value & Area Under Curve \\
\cline { 2 - 5 } & Unterococcus & 0.003 & 0.009 &
\end{tabular}

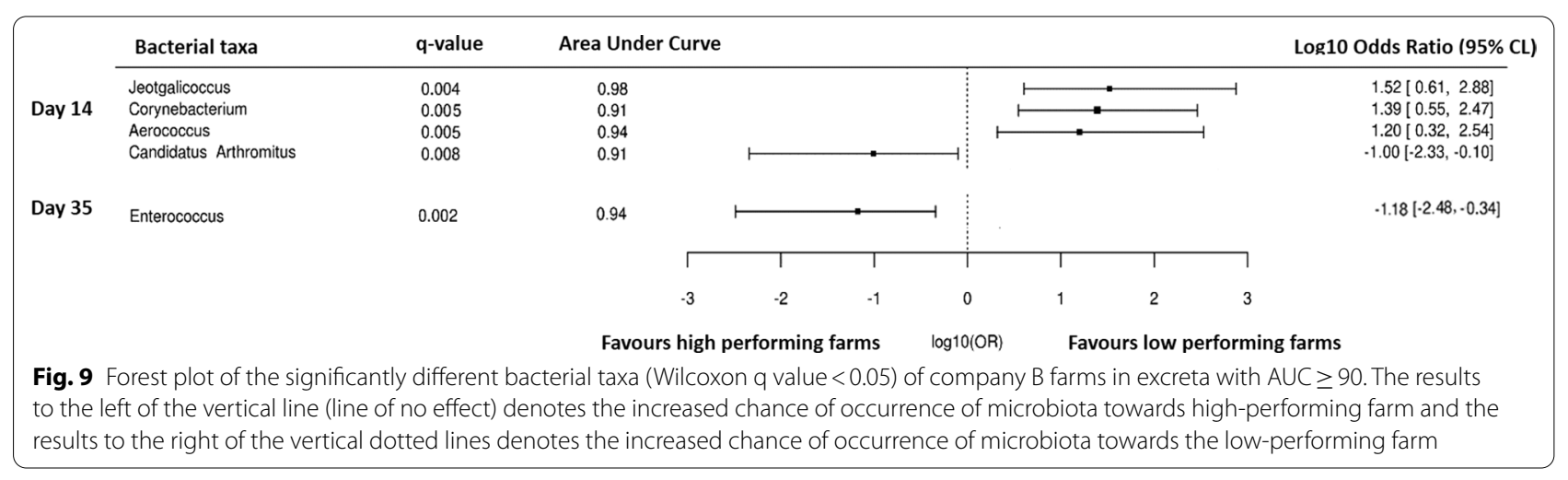


some bacterial taxa were associated with high or low performance, those signatures were highly dependent on the sample type, company, and the age of the bird.

The microbiota found within the dust of the poultry houses is presumably dependent largely on the excreta and litter microbiota with some influence of the environmental conditions on how those microbiota develop and spread over time. Excreta has been shown to form the major component of dust from turkey houses [25] and broiler chicken houses [26] and Luiken et al. [27] recently showed that poultry dust bacteriome are more diverse but associated with excreta bacteriome composition. Therefore, it is not surprising that $81 \%$ of bacterial taxa found in excreta samples were also found in dust. It is possible, however, that the different DNA extraction techniques used in this study could have influenced the detection of bacterial taxa in both sample types.

The microbial community composition varied between companies, with companies A and B sharing $55 \%$ of bacterial taxa in dust and $52 \%$ in excreta. This was expected as each integrator company used a different chicken breed (Cobb and Ross) and breed has been shown to influence the establishment of gut microbiota [28]. Other factors such as differences in feed formulation, hatchery, housing, bedding material, environment, and management factors within each company are also likely to have driven the observed quantitative differences in the microbial communities $[4,29]$.

Age also had a significant effect on microbial communities in dust and excreta in both companies, which is similar to previous findings [30-32]. It has been reported that bacterial richness increases during the first weeks of life while the variation in the microbiota of the gut decreases as the age advances [4]. From day 7-35, Weissella and Lactobacillus were the most dominant taxa in company A and Lactobacillus was the most dominant taxon in company B. This is in consistent with previous studies which showed that Lactobacillus was the predominant taxon in excreta $[12,33]$.

Farm performance explained the least variation in the bacterial community structure in both sample types, however, some specific bacterial taxa were differentially abundant in high and low performing farms in both dust and excreta samples. These included Enterococcus, Dickeya, Candidatus Arthromitus and an unclassified group of bacterial taxa.

Enterococcus, which was found as a bacterial signature of high-performing farms in this study, has already been explored for probiotic application in poultry. Enterococcus spp. are polysaccharide-degrading bacteria mainly responsible for degradation of mixed linked $\beta$-glucan in the intestine of meat chickens [34]. The combination of Enterococcus with Lactobacillus, Bifidobacterium, and
Streptococcus spp. have been used as probiotics and shown to have growth-promoting effects comparable to avilamycin treatment [35]. Candidatus Arthromitus has been shown to be associated with postnatal maturation of immune function in the mouse gut [36]. The functional roles of genus assigned to unclassified group would need further investigation although this is difficult to perform because of the lack of a system to study their functional role and characteristics [37]. In this study, Dickeya spp, which is an economically significant important crop pathogen [38], was also found to be associated with high performing farms. This is probably a coincidental result that needs further investigation.

In this study, no poultry pathogenic bacterial taxa was identified, however, Nocardia, Aerococcus and Corynebacterium found in low-performing farms could potentially cause disease in chickens. Previous research has shown that experimental inoculation of Nocardia asteroides or Nocardia transvalensis in 10-day-old cockerels resulted in depression, gasping and emaciation [39]. Aerococcus viridans has been isolated in meat chickens with hepatitis [40] and Corynebacteria may cause diphtheria [41]. Brachybacterium, Brevibacterium and Jeotgalicoccus which were present mostly in low-performing farms at higher levels require further investigation for their role in causing dysbiosis or subclinical infection in meat chickens. In humans, Brevibacterium spp was considered as an opportunistic cause of blood and cardiovascular infection in the immunocompromised humans [42], Brachybacterium nesterenkovii has been isolated in the blood stream infections and root canal infection in humans [43,44], so they may have some negative consequences when colonizing chickens. It is important to note that the detected taxa are dependent on relative abundances since the measurement and analysis is compositional by nature. This means that non-relevant changes in taxa abundances will also change the relative abundance of the identified taxon [45]. Thus, it is advisable to further evaluate the identified taxon related to high and low-performing farms more thoroughly via quantitative PCR in future studies.

In conclusion, the study identified microbial taxa present in poultry dust and excreta and their association with high and low-performance at flock-level. Such easily collected samples are a useful research tool for evaluating microbiota composition longitudinally in commercial flocks to find microbial signatures that correlate with flock health and productivity status. Examining multiple production cycles from the same farms and additional integrator companies is necessary to evaluate the reproducibility of the findings in this study. If the specific bacterial taxa associate with flock productive performance would be reproducible, this would assist in 
the development of a PCR panel for specific microbiota to evaluate and perhaps predict flock productive performance or in the evaluation of management intervention targeting gut health.

\section{Abbreviations}

AUC: Area under curve; DNA: Deoxyribonucleic acid; PCoA: Principal-coordinate analysis; PERMDISP: Permutational analyses of multivariate dispersions; PERMANOVA: Permutational multivariate analysis of variance; $16 S$ rRNA: $16 S$ ribosomal ribonucleic acid.

\section{Supplementary Information}

The online version contains supplementary material available at https://doi. org/10.1186/s42523-021-00127-y.

Additional file 1. Details of meat chicken farms used in this study. Farms A1 to A4 belong to company A and Farms B1 to B4 belong to company B.

Additional file 2. Permutational multivariate analysis of variance (PERMANOVA) results showing the influence of sample type, company, age, performance and their interaction on the bacterial community structure. $D f=$ degrees of freedom, $R^{2}=$ proportion of the variance explained by each model, PERMDISP = permutational analyses of multivariate dispersion.

Additional file 3. Principal-coordinate analysis plot using Bray-Curtis dissimilarity showing variation in the bacterial community structure by farm performance (high vs low), bird age (7, 14, 21, 28 and 35), sample type (dust and excreta) and company (A and B).

Additional file 4. Taxonomic assignment of the bacteria at phylum level in dust and excreta stratified by company.

Additional file 5. Bacteria taxa (genus level) that are shared between dust and excreta and unique to dust and excreta in company A farms.

Additional file 6 . Bacteria taxa (genus level) that are shared between dust and excreta and unique to dust and excreta in company $B$ farms.

Additional file 7. Distinguishing taxa between dust and excreta stratified by company. Linear discriminant analysis effect size was performed on the top 50 most abundant bacterial taxa (genus level) across all ages.

Additional file 8. Bacteria taxa (genus level) that are shared between company $\mathrm{A}$ and $\mathrm{B}$ farms and UNIQUE to company $\mathrm{A}$ and $\mathrm{B}$ farms in dust.

Additional file 9. Bacteria taxa (genus level) that are shared between company $A$ and $B$ farms and UNIQUE to company $A$ and $B$ farms in excreta.

Additional file 10. Distinguishing taxa between companies ( $\mathrm{A}$ and $\mathrm{B}$ ) stratified by sample type (dust and excreta). Linear discriminant analysis effect size was performed on the top 50 most abundant bacterial taxa (genus level) across all ages.

Additional file 11. Genera that were significantly different between high and low-performance farms of company $A$ in dust samples. The results are based on differences of mean abundance tested with Wilcoxon rank-sum test. P-values are corrected with false discovery rate (q-value).

Additional file 12. Genera that were significantly different between high and low-performance farms of company B in dust samples. The results are based on differences of mean abundance tested with Wilcoxon rank-sum test. P-values are corrected with false discovery rate (q-value).

Additional file 13. Genera that were significantly different between high and low-performance farms of company $A$ in pooled excreta samples. The results are based on differences of mean abundance tested with Wilcoxon ranksum test. P-values are corrected with false discovery rate (q-value).

Additional file $\mathbf{1 4}$. Genera that were significantly different between high and low-performance farms of company B in pooled excreta samples. The results are based on differences of mean abundance tested with Wilcoxon ranksum test. P-values are corrected with false discovery rate (q-value).

\section{Acknowledgements}

We are thankful to Danielle Smith and Sarbast Qassim for assistance during DNA extraction of samples. We are thankful to the veterinarians and farmers who participated in this study.

\section{Authors' contributions}

Conceptualization: PFG, RJM and SWW-B. Formal analysis: YRB, PFG and RJM. Methodology: TTHV and YRB. Writing original draft: YRB and PFG. Review and editing: YRB, RJM, TTHV, SWW-B and PFG. Supervision: PFG. All authors read and approved the final manuscript.

\section{Funding}

This study funded by AgriFutures Australia (Grant PRJ-011908).

\section{Availability of data and materials}

All the data related to this article were presented inside the manuscript and supplementary files. The sequence data used for analysis is available in NCBI under BioProject accession number PRJNA730489.

\section{Declarations}

Ethics approval and consent to participate

Not applicable.

\section{Consent for publication}

Not applicable.

\section{Competing interests}

The authors declare that they have no competing interest.

\section{Author details}

${ }^{1}$ Animal Science, School of Environmental and Rural Science, University of New England, Armidale, NSW 2351, Australia. ${ }^{2}$ School of Science, RMIT University, Bundoora West Campus, Plenty Rd, Bundoora, VIC 3083, Australia.

Received: 18 February 2021 Accepted: 13 September 2021

Published online: 02 October 2021

\section{References}

1. Sekirov I, Russell SL, Antunes LCM, Finlay BB. Gut microbiota in health and disease. Physiol Rev. 2010;90(3):859-904.

2. Apajalahti J, Kettunen A, Graham H. Characteristics of the gastrointestinal microbial communities, with special reference to the chicken. Worlds Poult Sci J. 2004;60(2):223-32.

3. Pan D, Yu Z. Intestinal microbiome of poultry and its interaction with host and diet. Gut microbes. 2014;5(1):108-19.

4. Kers JG, Velkers FC, Fischer EA, Hermes GD, Stegeman JA, Smidt H. Host and environmental factors affecting the intestinal microbiota in chickens. Front Microbiol. 2018:9:235

5. Celi P, Verlhac V, Calvo EP, Schmeisser J, Kluenter A-M. Biomarkers of gastrointestinal functionality in animal nutrition and health. Anim Feed Sci Technol. 2019;250:9-31.

6. Singh KM, Shah T, Deshpande S, Jakhesara SJ, Koringa PG, Rank DN, Joshi CG. High through put 165 rRNA gene-based pyrosequencing analysis of the fecal microbiota of high FCR and low FCR broiler growers. Mol Biol Rep. 2012;39(12):10595-602.

7. Torok VA, Allison GE, Percy NJ, Ophel-Keller K, Hughes RJ. Influence of antimicrobial feed additives on broiler commensal posthatch gut microbiota development and performance. Appl Environ Microbiol. 2011;77(10):3380-90.

8. Stanley D, Denman SE, Hughes RJ, Geier MS, Crowley TM, Chen H, Haring VR, Moore RJ. Intestinal microbiota associated with differential 
feed conversion efficiency in chickens. Appl Microbiol Biotechnol. 2012;96(5):1361-9.

9. Stanley D, Geier MS, Denman SE, Haring VR, Crowley TM, Hughes RJ, Moore RJ. Identification of chicken intestinal microbiota correlated with the efficiency of energy extraction from feed. Vet Microbiol. 2013;164(1-2):85-92.

10. Donaldson EE, Stanley D, Hughes RJ, Moore RJ. The time-course of broiler intestinal microbiota development after administration of cecal contents to incubating eggs. PeerJ. 2017;5:e3587.

11. Kers JG, Fischer EA, Stegeman JA, Smidt H, Velkers FC. Comparison of different invasive and non-invasive methods to characterize intestinal microbiota throughout a production cycle of broiler chickens. Microorganisms. 2019;7(10):431.

12. Stanley D, Geier MS, Chen H, Hughes RJ, Moore RJ. Comparison of fecal and cecal microbiotas reveals qualitative similarities but quantitative differences. BMC Microbiol. 2015;15(1):51.

13. Pauwels J, Taminiau B, Janssens G, De Beenhouwer M, Delhalle L, Daube G, Coopman F. Cecal drop reflects the chickens' cecal microbiome, fecal drop does not. J Microbiol Methods. 2015;117:164-70.

14. Tran T, Yegoraw A, Assen A, Walkden-Brown S, Gerber P. Genomic stability for PCR detection of infectious laryngotracheitis virus and infectious bronchitis virus in poultry dust samples stored under different conditions. Avian Dis. 2020;64(4):565-70.

15. Bindari YR, Kheravii SK, Morton CL, Wu S-B, Walkden-Brown SW, Gerber PF. Molecular detection of Eimeria species and Clostridium perfringens in poultry dust and pooled excreta of commercial broiler chicken flocks differing in productive performance. Vet Parasitol. 2021;291:109361.

16. Videnska P, Smerkova K, Zwinsova B, Popovici V, Micenkova L, Sedlar K, Budinska E. Stool sampling and DNA isolation kits affect DNA quality and bacterial composition following $16 \mathrm{~S}$ rRNA gene sequencing using MiSeq Illumina platform. Sci Rep. 2019;9(1):1-14.

17. Knudsen BE, Bergmark L, Munk P, Lukjancenko O, Prieme A, Aarestrup FM, Pamp SJ. Impact of sample type and DNA isolation procedure on genomic inference of microbiome composition. mSystems. 2016;1 (5):e00095-e116.

18. Fadrosh DW, Ma B, Gajer P, Sengamalay N, Ott S, Brotman RM, Ravel J. An improved dual-indexing approach for multiplexed 16S rRNA gene sequencing on the Illumina MiSeq platform. Microbiome. 2014;2(1):6.

19. Bolyen E, Rideout JR, Dillon MR, Bokulich NA, Abnet CC, Al-Ghalith GA, Alexander H, Alm EJ, Arumugam M, Asnicar F. Reproducible, interactive, scalable and extensible microbiome data science using QIIME 2. Nat Biotechnol. 2019:37(8):852-7.

20. Rognes T, Flouri T, Nichols B, Quince C, Mahé F. VSEARCH: a versatile open source tool for metagenomics. PeerJ. 2016;4:e2584.

21. Zakrzewski M, Proietti C, Ellis JJ, Hasan S, Brion M-J, Berger B, Krause L. Calypso: a user-friendly web-server for mining and visualizing microbiome-environment interactions. Bioinformatics. 2017;33(5):782-3.

22. Clarke K, Gorley R. Getting started with PRIMER v7. PRIMER-E: Plymouth, Plymouth Marine Laboratory 2015:20.

23. Anderson MJ, Walsh DC. PERMANOVA, ANOSIM, and the Mantel test in the face of heterogeneous dispersions: What null hypothesis are you testing? Ecol Monogr. 2013;83(4):557-74.

24. Anderson MJ. Distance-based tests for homogeneity of multivariate dispersions. Biometrics. 2006;62(1):245-53.

25. Feddes JJR, Cook H, Zuidhof MJ. Characterization of airborne dust particles in turkey housing. Can Agric Eng. 1992;34(3):273-80.

26. Ahaduzzaman M, Milan L, Morton CL, Gerber PF, Walkden-Brown SW. Characterization of poultry house dust using chemometrics and scanning electron microscopy imaging. Poult Sci. 100(7):101188.

27. Luiken RE, Van Gompel L, Bossers A, Munk P, Joosten P, Hansen RB, Knudsen BE, García-Cobos S, Dewulf J, Aarestrup FM. Farm dust resistomes and bacterial microbiomes in European poultry and pig farms. Environ Int. 2020;143:105971.

28. Emam M, Mehrabani-Yeganeh $H$, Barjesteh N, Nikbakht G, ThompsonCrispi K, Charkhkar S, Mallard B. The influence of genetic background versus commercial breeding programs on chicken immunocompetence. Poult Sci. 2014;93(1):77-84.
29. Schreuder J, Velkers FC, Bouwstra RJ, Beerens N, Stegeman JA, de Boer WF, van Hooft P, Elbers AR, Bossers A, Jurburg SD. An observational field study of the cloacal microbiota in adult laying hens with and without access to an outdoor range. Animal Microbiome. 2020;2(1):1-11.

30. Gérard P, Brézillon C, Quéré F, Salmon A, Rabot S. Characterization of cecal microbiota and response to an orally administered lactobacillus probiotic strain in the broiler chicken. J Mol Microbiol Biotechnol. 2008;14(1-3):115-22.

31. Ballou AL, Ali RA, Mendoza MA, Ellis J, Hassan HM, Croom WJ, Koci MD. Development of the chick microbiome: how early exposure influences future microbial diversity. Front Vet Sci. 2016;3:2.

32. Danzeisen JL, Kim HB, Isaacson RE, Tu ZJ, Johnson TJ. Modulations of the chicken cecal microbiome and metagenome in response to anticoccidial and growth promoter treatment. PLOS ONE. 2011;6(11):e27949.

33. Kaakoush NO, Sodhi N, Chenu JW, Cox JM, Riordan SM, Mitchell HM. The interplay between Campylobacter and Helicobacter species and other gastrointestinal microbiota of commercial broiler chickens. Gut Pathog. 2014;6(1):1-10

34. Shin M, Han S, Ji A, Kim K, Lee W. Isolation and characterization of bacteriocin-producing bacteria from the gastrointestinal tract of broiler chickens for probiotic use. J Appl Microbiol. 2008;105(6):2203-12.

35. Mountzouris K, Tsirtsikos P, Kalamara E, Nitsch S, Schatzmayr G, Fegeros K. Evaluation of the efficacy of a probiotic containing Lactobacillus, Bifidobacterium, Enterococcus, and Pediococcus strains in promoting broiler performance and modulating cecal microflora composition and metabolic activities. Poult Sci. 2007;86(2):309-17.

36. Bolotin A, De Wouters T, Schnupf P, Bouchier C, Loux V, Rhimi M, Jamet A, Dervyn R, Boudebbouze S, Blottière HM. Genome sequence of "Candidatus Arthromitus"sp. strain SFB-mouse-NL, a commensal bacterium with a key role in postnatal maturation of gut immune functions. Genome Announc. 2014;2(4):e00705-14.

37. Furtak K, Grządziel J, Gałązka A, Niedźwiecki J. Prevalence of unclassified bacteria in the soil bacterial community from floodplain meadows (fluvisols) under simulated flood conditions revealed by a metataxonomic approachss. CATENA. 2020;188:104448.

38. Rigault M, Buellet A, Masclaux-Daubresse C, Fagard M, Chardon F, Dellagi A. Quantitative methods to assess differential susceptibility of Arabidopsis thaliana natural accessions to Dickeya dadantii. Front Plant Sci. 2017;8:394.

39. Okoye J, Gugnani H, Okeke C. Experimental infection of chickens with Nocardia asteroides and Nocardia transvalensis. Avian Pathol. 1991;20(1):17-24.

40. Hariharan H, Oliveira S, Sharma S. Isolation of Aerococcus viridans from an outbreak of hepatitis in a broiler flock in Grenada. West Indian Vet J. 2008;2008:72-6.

41. Enurah L, Olubade T, Nwamo A, Sadiku R: an outbreak of Corynebacterium diphtheriae infection in broiler chickens in Lagos, Nigeria. Global J Med Res G Vet Sci Vet Med. 2016, 16(1).

42. Shweta F, Gurram PR, O'Horo JC, Khalil S: Brevibacterium species: an emerging opportunistic cause of bloodstream infections. In: Mayo clinic proceedings: 2021. Elsevier, pp. 1093-94.

43. Siqueira J Jr, Rôças I, Paiva S, Magalhães K, Guimarães-Pinto T. Cultivable bacteria in infected root canals as identified by $16 \mathrm{~S}$ rRNA gene sequencing. Oral Microbiol Immunol. 2007;22(4):266-71.

44. Brummaier T, Hinfothong P, Soe N, Tongmanakit J, Watthanaworawit W, Ling C. Brachybacterium nesterenkovii isolated from a human blood culture - a first report. New Microbes New Infect. 2020;36:100699.

45. Barlow JT, Bogatyrev SR, Ismagilov RF. A quantitative sequencing framework for absolute abundance measurements of mucosal and lumenal microbial communities. Nat Commun. 2020;11(1):1-13.

\section{Publisher's Note}

Springer Nature remains neutral with regard to jurisdictional claims in published maps and institutional affiliations. 